\title{
Intraoperative Scoring Assessment as a Prognostic Tool in Covid- 19 Associated Mucormycosis: A Short-Term Observational Study
}

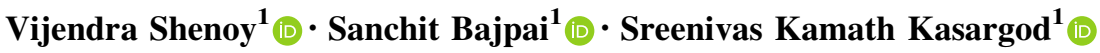

Received: 17 August 2021/Accepted: 28 November 2021/Published online: 14 January 2022

(C) Association of Otolaryngologists of India 2021

\begin{abstract}
To introduce an intraoperative scoring system for covid-19 associatedmucormycosis An observational study conducted among 43 cases of confirmed CAM whichincluded 35 males and 8 females, with an average age of 56 years. The surgicalapproach adopted in our cases included endoscopic surgical debridement withDenker's approach including mandatory pterygopalatine and infratemporal fossaexploration. All cases were intraoperatively scored using our designed intraoperativescoring assessment tool for mucormycosis. Postoperatively patient recovery wasassessed using $\mathrm{C}$ reactive protein levels and weekly imaging. Although an early observation in the post op period we observed highermortality among cases reporting with high scores as per our intraoperative reportingsystem. At the end of 2 months of completed treatment we report 6 cases of mortalityamong whom 5 cases were found to have scores $(>25)$ and one reported with a scoreof 18. This assessment helped us in grading the disease severity and also gaveus an insight about the postoperative prognosis too. Global scientific collaboration andreporting of a validated tool for CAM is of paramount importance to increase theknowledge with regard to this emerging disease.
\end{abstract}

Vijendra Shenoy

drvijendras@gmail.com

Sanchit Bajpai

dr.sanchitbajpai@gmail.com

Sreenivas Kamath Kasargod

ksreenivask77@gmail.com

1 Department of ENT and Head and Neck Surgery, Kasturba Medical College, Mangalore, Manipal Academy of Higher Education, Mangalore, Karnataka, India
Keywords Covid-19 - Mucormycosis · Fungal sinusitis · CAM

\section{Introduction}

COVID-19 associated mucormycosis (CAM) is an uncommon, acute, and aggressive fungal infection occurring in several immunocompromised states. CAM is seen to be associated with a very high residual morbidity and mortality due to the angioinvasive property of the fungus, thereby causing vascular occlusion and consequently resulting in extensive tissue necrosis [1].

Mucormycosis causes blood vessel thrombosis and resultant necrosis with "dry gangrene "causing the drugs to become non penetrable and lacking efficacy. Therefore, debridement of the necrotic tissues may be a critical step aiding in complete eradication of the disease. However, the timing and extent of surgical debridement necessary to maximize outcomes of mucormycosis has never been clearly defined.

The pterygopalatine fossa (PPF) is a small inverted fat filled area in the deep face which acts as a relaying station for various important neurovascular structures. It thus acts as an important source contributing to the rapid spread of infection and inflammation to the vital areas lying in its vicinity [2].

The PPF is considered to be a way of mucor spreads to retro-global space of orbit and infratemporal space. The orbit is accessible through the thin lamina papyracea of the ethmoid bone, infratemporal fossa, inferior orbital fissure, or orbital apex [3]. The cribriform plate of ethmoid, supraorbital fissure, and perineural invasion are potential gateways to intracranial extension. Extension into the PPF 
and ITF require rapid surgical debridement in addition to aggressive antifungal therapy [4].

Rhinocerebral mucormycosis usually presents as noticeable blackish brown necrosed tissue over the nasal mucosa, palate, skin, orbit which is either evident clinically or radiologically [5].

Due to the anatomic complexity of this small fat filled space, there is not enough evidence explaining its involvement despite having no clinical and radiological points of evidence for its involvement.

Pterygoid fossa exploration is thus regarded as an essential step intraoperatively, as it may be the hidden source harbouring the infection, which might deceive the operating surgeon leading to high post op recurrences and poor prognosis [6].

Since the past 6 months (January to June 2021) we have operated upon 43 of CAM and we aim to communicate our experience with the pre-operative imaging and the intraoperative findings among these cases. We also want to introduce a reporting system based on the intraoperative finding which could aid for assessment of prognosis of patient in the postoperative period.

\section{Materials And Methods}

Among all 43 cases, we found a male predominance (35 were males and 8 were females). The average age on presentation of patients was 56 years (28-80 years).

All patients were subjected to MRI with contrast imaging to see for disease extension and planning management. MRI T1 images showed isointense lesions [7] relative to brain in most cases and $\mathrm{T} 1 \mathrm{C}+(\mathrm{Gd})$ contrast images showed devitalised mucosa (black turbinate sign) [8] and also helped us in mapping out the disease extension and deciding the approach for their further management.

All the subjects who were suspicious for mucormycosis on clinical and radiological grounds were taken up for surgical debridement. In order to plan a complete clearance intraoperatively, we have designed a method for intraoperative scoring assessment (Fig. 1). Through this assessment we made it a practice to mandatorily explore all areas of potential concerns such as the pterygopalatine fossa and infratemporal fossa in all the cases with CAM, even in those not having any evidence of disease radiologically. The goal of surgical debridement was (1) Disease control (2) Obtaining a histopathology and aiding in (3) Microbiological diagnostics.

The surgical approach adopted in our cases included endoscopic Denker's approach with pterygopalatine and infratemporal fossa exploration. Endoscopic orbital decompression was done selectively in patients having orbital involvement (Fig. 2). Total maxillectomy was chosen modality of treatment in patients with extensive disease which was not accessible endoscopically.

Postoperatively patient recovery was assessed using $\mathrm{C}$ reactive protein levels and failure of the treatment was considered when CRP was elevated consecutively for 4 days which was further confirmed with postoperative imaging. All our patients were started on Inj liposomal amphotericin B $(5-10 \mathrm{mg} / \mathrm{kg})$ and later were continued on oral Posaconazole (600 mg on day 1 and then $300 \mathrm{mg}$ per day from day 2) as per their disease remission [9] with weekly assessment through imaging and alternate day renal function tests.

\section{Results And Analysis}

All 43 cases included in our study were confirmed with covid 19 associated mucormycosis. The average age on presentation of patients was 56 years (28-80 years).

Out of the 43 cases included in our study ( 35 were males and 8 were females).

Each patient was assessed separately and each site of involvement was scored intraoperatively. The total intraoperative score was calculated out of 45 which was the maximum score as per our scoring system.

The outcome was assessed by calculating CAM associated mortality post operatively and their intraoperative scores were compared (Fig. 3).

The cut off score in our study was set as 25 as per authors discretion as statistical analysis for cut off calculation couldn't be performed due to limited sample size.

Although an Early Observation in the Post op Period we Observed Higher Mortality Among Cases Reporting with High Scores as per our Intraoperative Reporting System

At the end of 2 months of completed treatment we report 6 cases of mortality among whom 5 cases were found to have scores $(>25)$ and one reported with a score of 18 .

We also observed that patients with higher scores $(>25)$ were mostly males $14.3 \%(5 / 35)$ and 1 female (12.5\%).

Among the 6 patients we observed mortality with high intraoperative scores, 4 patients underwent endoscopic Denker's approach with pterygopalatine and infratemporal fossa clearance including endoscopic orbital decompression. We also observed that these patients had postoperative disease recurrence and required re-debridement which could have been a contributing factor for mortality. 2 out of 6 patients with high scores underwent total maxillectomy without orbital exenteration.

An interesting observation made in our study was despite having no radiological evidence of disease extension in pterygopalatine and infratemporal fossa, we found disease extension in pterygopalatine fossa among $37.2 \%$ 
Fig. 1 Intraoperative Scoring Assessment

\begin{tabular}{|c|c|c|}
\hline SINUS INVOLVEMENT & $\underline{\text { RIGHT SINUS }}$ & $\underline{\text { LEFT SINUS }}$ \\
\hline Maxillary & $0-2$ & $0-2$ \\
\hline Ethmoid & $0-2$ & $0-2$ \\
\hline Sphenoid & $0-2$ & $0-2$ \\
\hline Frontal & $0-2$ & $0-2$ \\
\hline \multicolumn{3}{|c|}{ ( 0 - no involvement, 1 - mucosal involvement, 2 - bone involvement) } \\
\hline \multicolumn{3}{|l|}{ NASAL CAVITY } \\
\hline Septum & \multicolumn{2}{|l|}{$0-2$} \\
\hline Middle turbinate & \multicolumn{2}{|l|}{$0-2$} \\
\hline Inferior turbinate & \multicolumn{2}{|l|}{$0-2$} \\
\hline ( 0 - no involvement, 1 - mucosal involvement, 2 - bone involvement $)$ & \multicolumn{2}{|c|}{$0-2$} \\
\hline \multicolumn{3}{|c|}{$\begin{array}{l}(0 \text { - no involvement, } 1 \text { - mucosal involvement, } 2 \text { - bone involvement, } \\
3 \text { - intracranial extension) }\end{array}$} \\
\hline \multicolumn{3}{|c|}{$\begin{array}{l}\text { ORBITAL INVOLVEMENT } \\
\\
(0-\text { no involvement, } 1 \text { - lamina papyracea: extraconal involvement, } \\
2 \text { - intraconal involvement) }\end{array}$} \\
\hline \multicolumn{3}{|c|}{ PTERYGOPALATINE FOSSA INVOLVEMENT } \\
\hline \multicolumn{3}{|c|}{$(0-$ no involvement, 2 - involvement seen $)$} \\
\hline$(0-$ no involvement, 2 - involvement seen - thrombosed/necrosed $)$ & \multicolumn{2}{|l|}{$0-2$} \\
\hline \multicolumn{3}{|c|}{ ( 0 - no involvement, 1 - inflamed, 2 - necrosed) } \\
\hline \multicolumn{3}{|c|}{$(0-$ no involvement, $2-$ necrosed $)$} \\
\hline \multicolumn{3}{|c|}{ INFRATEMPORAL FOSSA INVOLVEMENT } \\
\hline$(0-$ no involvem ent, 2 - involvement seen $)$ & \multicolumn{2}{|l|}{$0-2$} \\
\hline \multicolumn{3}{|c|}{$(0-$ no involvement, 2 - involvem ent seen - thrombosed/necrosed $)$} \\
\hline $\begin{array}{l}\text { Neu ral - compartment } \\
(0-\text { no involvem ent, } 1-\text { in } t\end{array}$ & d, 2 - necrosed) & $0-2$ \\
\hline $\begin{array}{l}\text { Muscular compartment } \\
(0-\text { no involvement, } 2-\text { necr }\end{array}$ & $0-2$ & \\
\hline
\end{tabular}




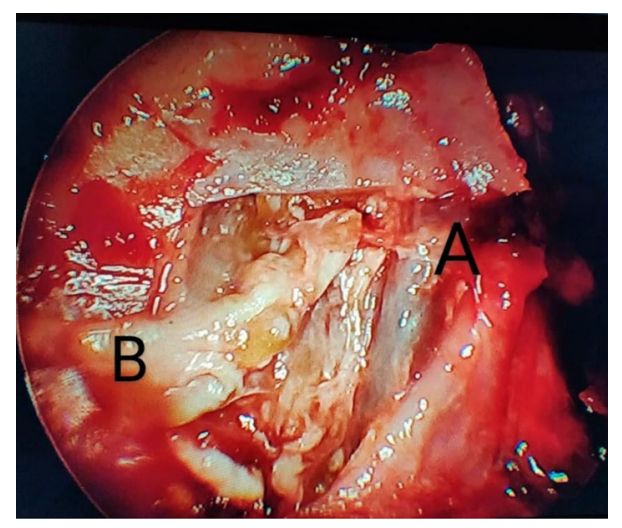

Fig. 2 Cam Showing Involvement Of Pterygopalatine Fossa

cases (16/43) and infratemporal fossa involvement among $20.9 \%(9 / 43)$ cases. In such circumstances the intraoperative scores were found to much higher in these cases despite having no radiological evidence of disease in these keystone areas.

\section{Discussion}

Mucormycosis is a life-threatening infection caused by fungi of the order Mucorales. Mucormycosis of the orbit is a vision-threatening and potentially fatal infection resulting in angioinvasion, thrombosis, and ischemic necrosis of tissues. Mucor, Rhizopus, Apophysomyces, Cunninghumella are the common organisms responsible for the infection $[10,11]$. Similar study was conducted previously by Shah and Dave et al. [10] who devised a scoring system with a cut off score of 23 (as per authors discretion) that predicts the stage at which the exenteration needs to be carried out among 15 patients with mucormycosis. Our study includes an intraoperative assessment scoring system with a cut off score of 25 in which we have scored each subsite in an extensive manner which can aid the surgeon in assessing disease severity and predict treatment outcomes in patients with CAM. For decades, the mortality rate of mucormycosis has remained $\geq 40 \%$ despite aggressive treatment modalities [12]. There is no good staging system to categorize the disease entity. On the basis of signs and symptoms the disease process can be grouped into 3 stages which include sinonasal disease, rhino-orbital disease, and rhino-orbital cerebral disease [13].

Meticulous physical examination including examination of oral cavity, eye, and cranial nerves is very important for diagnosing and assessing extent as the radiological findings are non-specific especially in the early stages [14].

Surgical removal of all the necrotic tissue is the most important factor in the treatment of ROCM. But no clear guidelines are present with regards to it. As the pterygopalatine fissure is the main reservoir for Mucor, the posterior wall of the maxillary sinus, even if intact, must always be removed irrespective of the approach or the extension of the surgery [15].

Necrosis is not always present in the orbital tissue in ROM. It may be due to spill over inflammation from the adjacent paranasal sinuses or Mucor cellulitis. Hence, "a direct inspection of orbital contents, looking carefully for signs of necrosis", is critical in determining the need for orbital exenteration [16].

Thus, exploring all areas intraoperatively is a critical step for complete disease clearance and reducing chances of post- operative recurrence and mortality. We noticed in many of our cases the pterygopalatine fossa can act as a hidden area of concern for the fungus to grow despite having no clinical and radiological evidence of its involvement.

In order to achieve this goal, we have structured an intraoperative assessment scoring system through which we scored each of our patient diagnosed with CAM.
Fig. 3 Score Category Mortality Crosstabulation

\begin{tabular}{|c|c|c|c|c|c|}
\hline & \multirow{2}{*}{\multicolumn{2}{|c|}{ INTRAOPERATIVE SCORE }} & \multicolumn{2}{|c|}{ MORTALITY } & \multirow[b]{2}{*}{ Total } \\
\hline & & & Alive & Death & \\
\hline \multirow[t]{4}{*}{ Score category } & $<25$ & Count & 36 & 1 & 37 \\
\hline & & $\%$ Within Score category & $97.3 \%$ & $2.7 \%$ & $100.0 \%$ \\
\hline & $>=25$ & Count & 1 & 5 & 6 \\
\hline & & $\%$ Within Score category & $16.7 \%$ & $83.3 \%$ & $100.0 \%$ \\
\hline \multirow[t]{2}{*}{ Total } & & Count & 37 & 6 & 43 \\
\hline & & $\%$ Within Score category & $86.0 \%$ & $14.0 \%$ & $100.0 \%$ \\
\hline
\end{tabular}


Through this assessment tool we addressed and explored all critical areas intraoperatively and found 24 patients having hidden disease in the pterygopalatine and infratemporal fossa despite having a normal radiological picture.

This assessment helped us in grading the disease severity and also gave us an insight about the postoperative prognosis too. Although an early observation in the post op period we observed higher mortality (6 patients) among cases reporting with high scores as per our intraoperative reporting system.

Global scientific collaboration and reporting of new information related to this is of paramount importance to increase the knowledge with regard to the novel viral infection associated with mucormycosis. Hence, we request that this method of intraoperative reporting and scoring can be further standardized/modified/globally validated as a tool for prognosis assessment among cases of ROCM.

Author Contributions DR. Vijendra Shenoy DR. Sanchit Bajpai DR.Sreenivas Kamath Conceptualization, Methodology, Validation, formal analysis, data curation, original draft preparation, draft review, draft editing, Funding acquisition.

\section{Declarations}

Conflict of interest The authors declare that they have no conflict of interest.

\section{References}

1. Peterson KL, Wang M, Canalis RF, Abemayor E (1997) Rhinocerebral mucormycosis: evolution of the disease and treatment options. Laryngoscope 107(7):855-862

2. Hosseini SM, Borghei P (2005) Rhinocerebral mucormycosis: pathways of spread. Eur Arch Oto-Rhino-Laryngol Head Neck 262(11):932-938

3. Spellberg B, Ibrahim AS (2010) Recent advances in the treatment of mucormycosis. Curr Infect Dis Rep 12(6):423-429
4. Goyal P, Leung MK, Hwang PH (2009) Endoscopic approach to the infratemporal fossa for treatment of invasive fungal sinusitis. Am J Rhinol Allergy 23(1):100-104

5. Mane R, Patil B, Mohite A, Mohanty R (2019) Facial nerve palsy: an unusual presentation in patients with rhino cerebral mucormycosis. Ind J Otolaryngol Head Neck Surg 71(3):2110-2113

6. Chandramouli MP, Sankaranarayanan G, Prasanna RV (2018) Imaging in Rhino-OrbitaL Mucormycosis. J Evol Med Dental Sci-Jemds 7(11):1385-1390

7. Herrera DA, Dublin AB, Ormsby EL, Aminpour S, Howell LP (2009) Imaging findings of rhinocerebral mucormycosis. Skull Base 19(2):117

8. Safder S, Carpenter JS, Roberts TD, Bailey N (2010) The, "black turbinate" sign: an early MR imaging finding of nasal mucormycosis. Am J Neuroradiol 31(4):771-774

9. Vehreschild JJ, Vehreschild MJ, Mellinghoff SC, Vehreschild JJ. (2019) Global guideline for the diagnosis and management of mucormycosis: an initiative of the European confederation of medical mycology in cooperation with the mycoses study group education and research consortium.

10. Shah K, Dave V, Bradoo R, Shinde C, Prathibha M (2019) Orbital exenteration in rhino-orbito-cerebral mucormycosis: a prospective analytical study with scoring system. Ind J Otolaryngol Head Neck Surg 71(2):259-265

11. Lee AS, Lee PW, Allworth A, Smith T, Sullivan TJ (2020) Orbital mycoses in an adult subtropical population. Eye 34(9):1640-1647

12. Werthman-Ehrenreich A (2021) Mucormycosis with orbital compartment syndrome in a patient with COVID-19. Am J Emerg Med 1(42):264-e5

13. Mane RS, Watve JK, Mohite AA, Patil BC (2007) Rhinocerebral mucormycosis: a deadly disease on the rise. Ind J Otolaryngol Head Neck Surg 59(2):112-5

14. Hernández OP, Calleros HM, Daguerre GS, González AS (2015) Rhino-orbito-cerebral mucormycosis. Management strategies to avoid or limit intracraneal affection and improve survival. Acta Otorrinolaringologica 66(6):348-52

15. Gamaletsou MN, Sipsas NV, Roilides E, Walsh TJ (2012) Rhinoorbital-cerebral mucormycosis. Cur Infect Dis Rep 14(4):423-34

16. Avet PP, Kline LB, Sillers MJ (1999) Endoscopic sinus surgery in the management of mucormycosis. J neuro-ophthalmol: Off $\mathbf{J}$ North Am Neuro-Ophthalmol Soc 19(1):56-61

Publisher's Note Springer Nature remains neutral with regard to jurisdictional claims in published maps and institutional affiliations. 